\title{
On the computation of interface stresses by finite elements for sandwich materials
}

\author{
Vincent MANET \\ École des Mines de Saint-Etienne, Material and Mechanical Department, 158, \\ cours Fauriel, 42023 Saint-Etienne cedex 2, France
}

\begin{abstract}
In this article, we recall the methods generally followed to compute stresses, and then develop a simple alternative. In order to improve the accuracy and stability with very few elements, we improve the proposed method to a second one. The latter method is then assessed on two examples to compute interface stresses of a sandwich structure. The robustness of the method is terms of convergence speed, accuracy and stability is demonstrated. The simplicity of formulation and implementation is also emphasized.
\end{abstract}

Key words: sandwich structure, interface stresses, post-processing, local Reissner

\section{Introduction}

Progress in materials science have led to the industrial use of more and more new materials. Among these materials, sandwich materials only begin to make great strides and to be appreciated in the field of transport (automotive, aeronautics, shipbuilding and railroads) or civil engineering. Nevertheless, their numerical modelling still suffers from a lack of accuracy.

A sandwich structure is a 3-layer laminated one, whose layers have very different geometrical and mechanical properties:

- the skins, which are generally thins and very stiff, work in membrane;

- while the core, which is thick and has low stiffness and density, is subjected to shear efforts.

Every material which can be obtained in a thin skin shape is acceptable for the skins and every material with low density is acceptable for the core, so 
that sandwich materials cover an extremely wide domain, and are therefore difficult to study in a global way.

Nevertheless, whatever the chosen materials may be, we shall restrain our study to the following assumptions concerning the conditions for the physical existence of interfaces between the different layers:

- the displacement field must be continuous:

- the continuity of the normal component at the interface implies that there is no disbond;

- the continuity of the tangential component that there is no sliding;

- with these hypotheses on the displacements, the vector $\boldsymbol{T}\left(T_{i}=\sigma_{i j} n_{j}\right.$ is the normal trace of the stress tensor along a surface $(3 \mathrm{D})$ or a curve $(2 \mathrm{D})$ with outgoing normal vector of components $n_{j}$ ) must be continuous at interfaces too.

The values of interlaminar stresses (and especially of transverse shear stress, since the core is essentially subjected to shear loading) are of great importance for designers. Thus, it is to be noticed that it is necessary:

- to accept totally different mechanical and geometrical properties in each layer: this is the definition of a sandwich;

- to take effects of transverse shear into account: experiments show that sandwiches often fail in delamination, i.e. due to transverse shear loading. Numerically, this component can reach high values;

- to eliminate the plane stress assumption used in the classical laminate theory: it has been observed that the out-of-plane component (i.e. in the direction of the thickness) is not equal to zero. Even, if its value remains small compared to others components, it is sufficiently high in the core, where the rigidity in this direction is low, to induce important effects.

In this article, we do not treat failure modes, particular to sandwich structures, which are described by Teti and Caprino ${ }^{1}$ for example. We only study sandwich structures in the case of three-dimensional linear elasticity theory.

\section{$2 \quad$ Finite element modelling}

The problem is to ensure the continuity of only some components of the stress tensor at interfaces (the vector $\boldsymbol{T}$ ), so that the physical coherence of the interface and the force equilibrium state will be verified. 
First, we must remark that it is possible to use "special elements" for the computation of highly heterogeneous structures:

- mixed elements of Reissner's type, or hybrid mixed Pian and Tong based elements $^{2}$ : their main advantage is to directly yield the continuity of the stress tensor; their disadvantages are that all components of the stress tensor are continuous which is excessive;

- "interface elements" have been developed from Reissner's formulation, by relaxing superabundant continuities by removing excessive components (i.e. by adding conditions between components) or by moving some degrees of freedom to the interior of the element, so that they do not enter the assembly process. Such elements perfectly fit the requirements, but are extremely difficult to developp (it does not exist a systematic method to generate them). For these method, one can refer to the $\mathrm{PhD}$ thesis of Aivazzadeh ${ }^{3}$ for example;

- "hybrid sandwich elements", based on different functional modified using Lagrange multipliers have also been developed. They fit the requirements and are more easy to develop than the previous one (it can be done systemaically). Nevertheless, the global stiffness matrix is not always symetrical: see for example ${ }^{4,5}$.

All these special elements have the same main disadvantage: they lead to a stiffness matrix which is bigger (more variables than the displacement method since we add nodal stresses) and no more definite-positive, which means that the computation time is increased.

In order to use the most efficient algorithms, it is necessary to conserve a definite-positive stiffness matrix: in other words, it is more judicious to use a classical modelling using displacements elements and an efficient postprocessing method. In this case, it is therefore necessary to modify the way of deriving nodal stresses from nodal displacements in order to ensure the continuities and the accuracy.

When using classical (displacement) elements, it is not possible to reach directly the nodal stresses, i.e. nodal stresses have to be recovered from the nodal displacements using the following formula:

$$
\tau=N_{\sigma} D \mathcal{L} N_{u} q
$$

where $\boldsymbol{\tau}$ is the vector of nodal stresses, $\boldsymbol{N}_{\boldsymbol{\sigma}}$ and $\boldsymbol{N}_{\boldsymbol{u}}$ the matrices of shape functions for stresses and displacements respectively, $\boldsymbol{D}$ the generalized Hooke's matrix relating stresses to strains, $\mathcal{L}$ the matrix of the differential operator relating strains to displacements and $\boldsymbol{q}$ the vector of nodal displacements. 
However, the method corresponding to relation (1) has been shown to be numerically the worst ${ }^{6}$. Hence, instead of using this relation, finite elements programs generally minimize the difference between the direct computation and the mixed formulation (Reissner's solution).

It means that one tries to minimize the difference between nodal stresses computed from displacements:

$$
\sigma_{u}=D \mathcal{L} N_{u} q
$$

and stresses obtained in a "mixed" way:

$$
\sigma_{m}=N_{\sigma} \tau
$$

This minimization process is generally expressed using a least squares formulation ${ }^{6}$ :

$$
\int_{\Omega}\left(\boldsymbol{\sigma}_{\boldsymbol{m}}-\boldsymbol{\sigma}_{\boldsymbol{u}}\right)^{2} d \Omega=0
$$

where $\Omega$ is the studied volume (3D) or surface (2D), or using the "stress projection" $\operatorname{method}^{7}$ (also known as $\mathrm{L}^{2}$-projection):

$$
\int_{\Omega}{ }^{t} \boldsymbol{N}_{\boldsymbol{\sigma}}\left(\boldsymbol{\sigma}_{\boldsymbol{m}}-\boldsymbol{\sigma}_{\boldsymbol{u}}\right) d \Omega=0
$$

which yields a matrix system of the following form:

$$
M_{\sigma} \tau=P_{u}
$$

with:

and

$$
\boldsymbol{M}_{\boldsymbol{\sigma}}=\int_{\Omega}{ }^{t} \boldsymbol{N}_{\sigma} \boldsymbol{N}_{\boldsymbol{\sigma}} d \Omega
$$

$$
\boldsymbol{P}_{\boldsymbol{u}}=\int_{\Omega}{ }^{t} \boldsymbol{N}_{\boldsymbol{\sigma}} \boldsymbol{D} \mathcal{L} \boldsymbol{N}_{\boldsymbol{u}} \boldsymbol{q} d \Omega
$$

from which nodal stresses can be found as:

$$
\boldsymbol{\tau}=M_{\sigma}{ }^{-1} P_{u}
$$

In these equations, $\boldsymbol{N}_{\boldsymbol{\sigma}}=\boldsymbol{N}_{\boldsymbol{u}}$ is generally used.

It is to be noticed that the minimization process can be performed over the whole structure (global) or element by element (local), as explained in ${ }^{6}$. Nevertheless, Hinton and Campbell have shown that it does not worth it to do a global minimization (which implies a big matrice representing the whole structure), because the local process converges towards the same solution and only involves small matrices ${ }^{6}$.

It is also known that the displacements formulation together with, for example, a least squares method needs a very fine and regular mesh to converge towards the right value. 


\section{3 "direct local Reissner" method}

Taking the aformentioned points into account, we come to the conclusion that it would probably be simpler and faster to find the stress field using directly Reissner's formulation in each element, instead of minimizing a difference between two solutions.

Hence, the Reissner's formulation can be decoupled in order to be used as a post-processing method, and performed only locally. It this case, nodal stresses are computed from displacements using the following relation:

$$
\boldsymbol{\tau}=\boldsymbol{A}^{-1} \boldsymbol{B} \boldsymbol{q}
$$

with:

$$
\begin{aligned}
& \boldsymbol{A}=+\int_{\Omega}{ }^{t} \boldsymbol{N}_{\boldsymbol{\sigma}} \boldsymbol{S} \boldsymbol{N}_{\boldsymbol{\sigma}} d \Omega \\
& \boldsymbol{B}=+\int_{\Omega}{ }^{t} \boldsymbol{N}_{\boldsymbol{\sigma}} \mathcal{L} \boldsymbol{N}_{\boldsymbol{u}} d \Omega
\end{aligned}
$$

where $\boldsymbol{S}=\boldsymbol{D}^{-1}$ is the compliance matrix.

With such a formulation, we do not need any more to speak about "convergence", since we calculate Reissner's solution. We shall refer to this method as the "direct local Reissner" method.

\section{4 "local Reissner"}

All of the methods presented, i.e. least squares, stress projection or direct local Reissner, work perfectly and converge towards the Reissner solution, although not with the same speed. However, this was not studied.

An other problem appearing is the "averaging problem": with the methods presented above, nodal stresses are reached within each element. The results have then to be averaged at nodes belonging to more than one element. It can lead to very inaccurate results when the material properties varie from one element to an other (i.e. at an interface).

The present problem is to accuratly compute stresses at the bonded interface between two very different materials, and if possible to speed up the convergence compared to methods presented in the previous sections. 


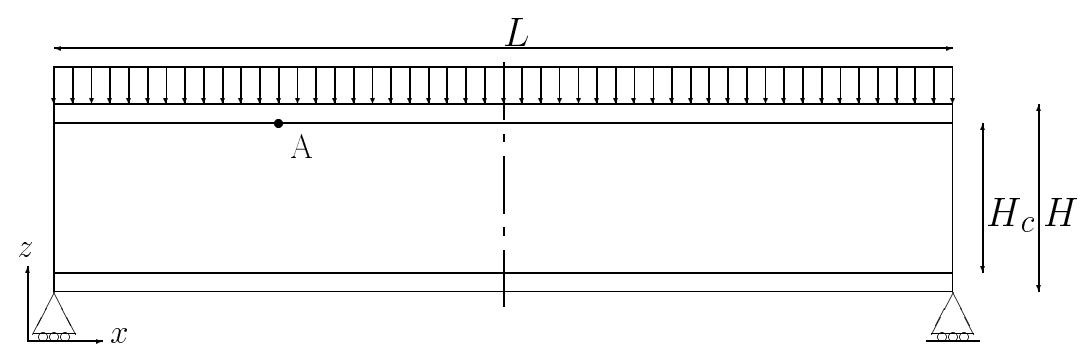

Fig. 1. Sandwich beam

The "averaging problem" emphazises the key of the problem: the choice of the volume or surface of interest, $\Omega$.

- if $\Omega$ is the whole structure, the process is global and involves a big matrice;

- if $\Omega$ is one single element, the process is local, but we face the averaging problem.

For this reason, we propose to use the formulation of the "direct local Reissner" method, but only where it is needed: this method is applied only locally, along the interfaces, on two adjacent elements (one belonging to the core, the other to a skin), so that we only deal with small matrices. So, $\Omega$ is the volume (or surface) represented by two elements on each side on the interface.

This method will be referred to as the "local Reissner" method.

We can already point out the mains features of the local Reissner method:

- we use a direct computation of stresses from displacements (not a minimization process);

- we directly reach nodal stresses (and not stresses located at Gauss points, from where there are extrapolated, and then averaged);

- we only have small matrices, which means that it becomes easy to program the method and to implement it in existing finite elements codes. Results presented in the next sections have been obtained with a macro command implemented in the FEM package ANsYS.

\section{Example 1: sandwich beam}

We now illustrate the effective improvement yielded by the local Reissner method.

Let us consider a simply supported sandwich beam subjected to an uniform pressure on its top face, as shown in figure 1. The skins are made of CFRP 


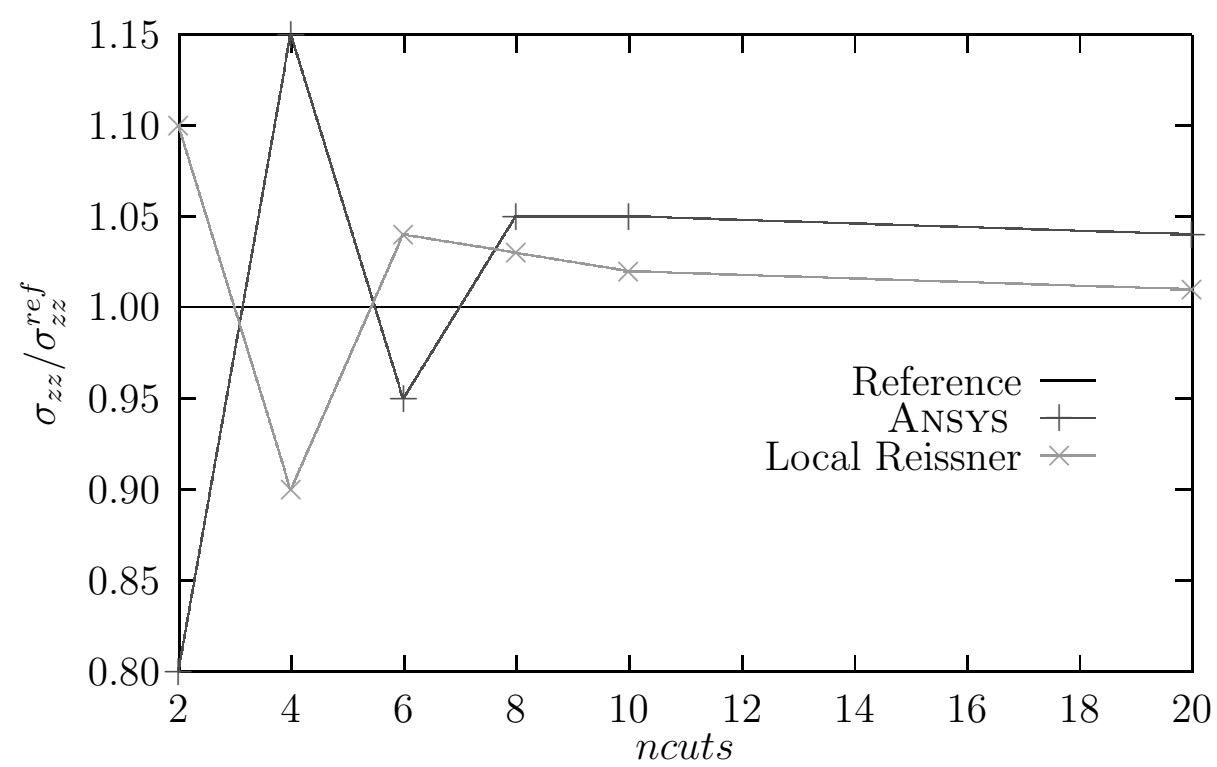

Fig. 2. Convergence of $\sigma_{z z}$

T300-914 $\left(E_{x x}=125 \mathrm{GPa}, E_{y y}=E_{z z}=10 \mathrm{GPa}, \nu_{x y}=\nu_{y z}=\nu_{x z}=0.3\right.$ (major Poisson ratios), $G_{x y}=G_{y z}=G_{x z}=5 \mathrm{GPa}$ ) and the core of foam $(E=340 \mathrm{MPa}$ and $\nu=0.40)$. The total length is $L=24 \mathrm{~mm}$ and the total height $H=2 \mathrm{~mm}$. The width is taken equal to $1 \mathrm{~mm}$. The core represents $80 \%$ of the total height of this symmetrical sandwich. The applied pressure is $q=-1 \mathrm{~N} / \mathrm{mm}^{2}$. By symmetry, only one half of the beam has been modelled.

Since it is known that the displacements method enables us taking this kind of materials into account, and can lead to an accurate representation of stresses, even at interfaces, if a very fine and regular mesh is used, our reference mesh is likely to be: 4 elements through the thickness of each skin, 32 through the thickness of the core, and 400 longitudinal cuts of the half beam, so 16000 elements will be used. It permits to reach Pagano's solution ${ }^{9}$.

The point of interest for our study is the point A shown on figure 1, at coordinate $x=L / 4$ and at the interface between the top skin and the core.

At this point, the quantities of interest are the components of stresses: interlaminar components $\sigma_{x z}$ and $\sigma_{z z}$ must be continuous, whereas the component $\sigma_{x x}$ does not have to satisfy this continuity (and in this case, must be discontinuous).

Convergence results concerning the interlaminar stresses $\sigma_{z z}$ and $\sigma_{x z}$ are illustrated in figures 2 and 3.

Only results corresponding to the later method, the local Reissner method, are presented. They are compared with results obtained with the well known 


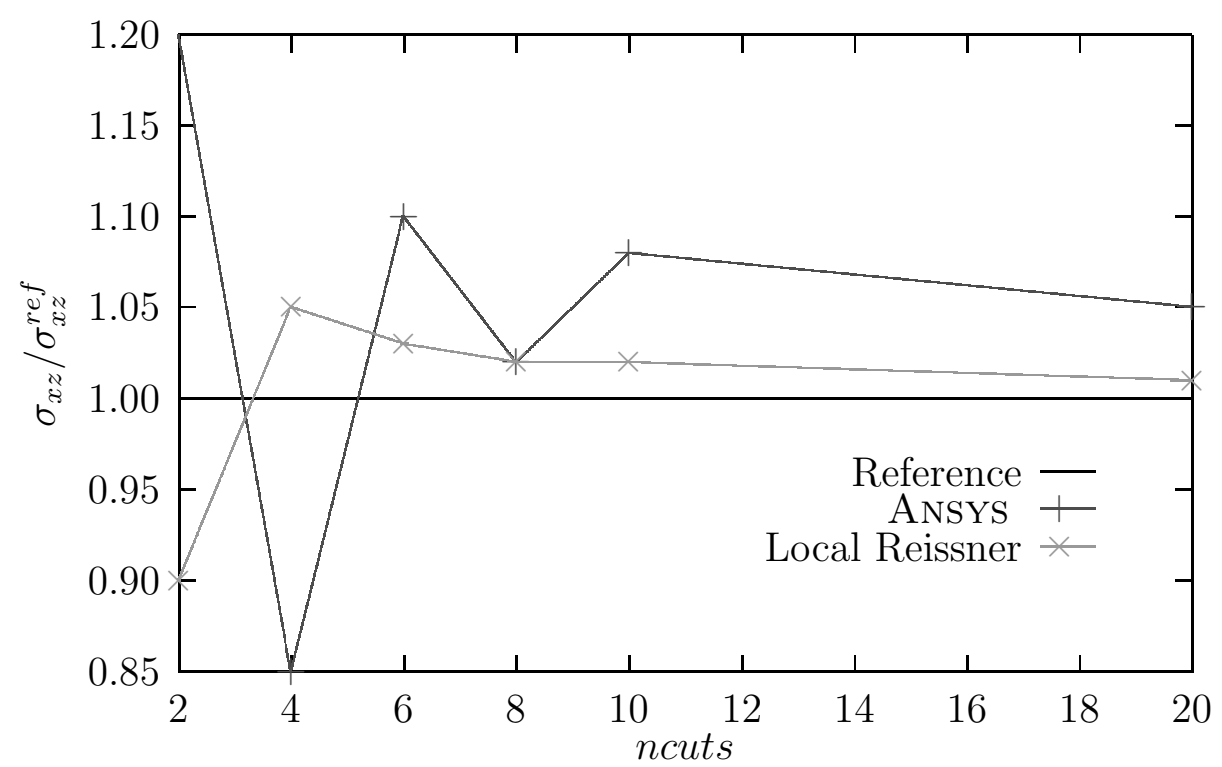

Fig. 3. Convergence of $\sigma_{x z}$

software ANSYs 5.3, which uses a least squares minimization in each element and nodal averaging to compute the nodal stresses from displacements.

Only a very coarse meshing is used for the following reasons:

- with such "limit cases" it is possible to see the stability and convergence speed of the methods;

- only very few elements permit to have a very good accuracy of displacements, one of the most important quantity in design. We shall use only 1 element through the thickness of each layer. Only the number of longitudinal cuts of the half beam, ncuts, will vary in the study (with only 2 cuts, the error concerning displacements is less than $2 \%$ );

- this case is simple. During the design stage, calculations are always effectuated using very few elements. But engineers hope (and it should be so) that it is sufficient to have a correct accuracy, even for stresses.

One important factor entering the accuracy of results is the ratio $E_{s} / E_{c}$ of Young modulus of the skins over Young modulus of the core, in the working direction (which depends on the chosen example).

Figure 4 illustrates the stability of the local Reissner when the $E_{s} / E_{c}$ increases. For this purpose, we plot the interlaminar shear stress $\sigma_{x z}$ as a function of the ratio $E_{s} / E_{c}$ on the presented sandwich beam, but composed of isotropic materials for the skins and the core.

Others examples, such as a sandwich plate or an adhesive joint, can be found in ${ }^{5}$ and ${ }^{8}$. 


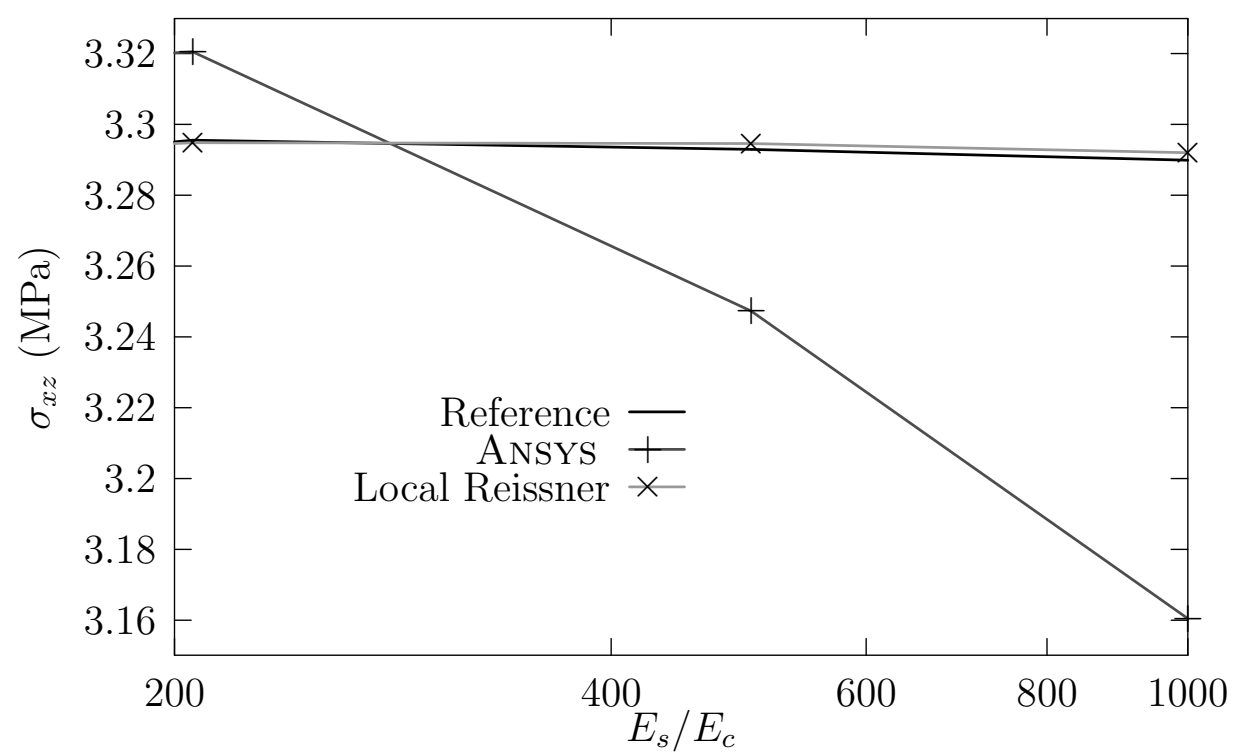

Fig. 4. $\sigma_{x z}$ at high $E_{s} / E_{c}$ ratios

To conclude this example, it is important to emphasize that with the utilized mesh the local Reissner method, in all cases:

- leads to more accurate results;

- increases the convergence speed;

which it is particularly important for designers who generally use relatively coarse meshes. In other words, the robustness of the local Reissner method has been demonstrated on this example.

\section{Example 2: U-type beam}

We shall now briefly focus on the stability of the local Reissner method. For this purpose, let us consider the U-type beam presented in figure 5 .

$H=2 \mathrm{~mm}$ and $L=20 \mathrm{~mm}$ are constants, but the radius $R_{\text {int }}$ varies from $5 \mathrm{~mm}$ to $0.125 \mathrm{~mm}$ (leading to a ratio $R / H$ between 3 and 0.5625 ). This is the important point of the study since we know that decreasing this ratio is equivalent to getting closer to a singularity.

Once again, the core represents $80 \%$ of the total height of this symmetrical sandwich. But isotropic materials are used (to simplify the interpretation of results). The skins are made of aluminum $\left(E_{s}=70000 \mathrm{MPa}\right.$ and $\left.\nu_{s}=0.34\right)$ and the material of the core varies : $\nu_{c}=0.40$ is constant, but $E_{c}$ takes the following values: $340 \mathrm{MPa}$ (epoxy), $34 \mathrm{MPa}$ (foam), $140 \mathrm{MPa}$ (foam) and 


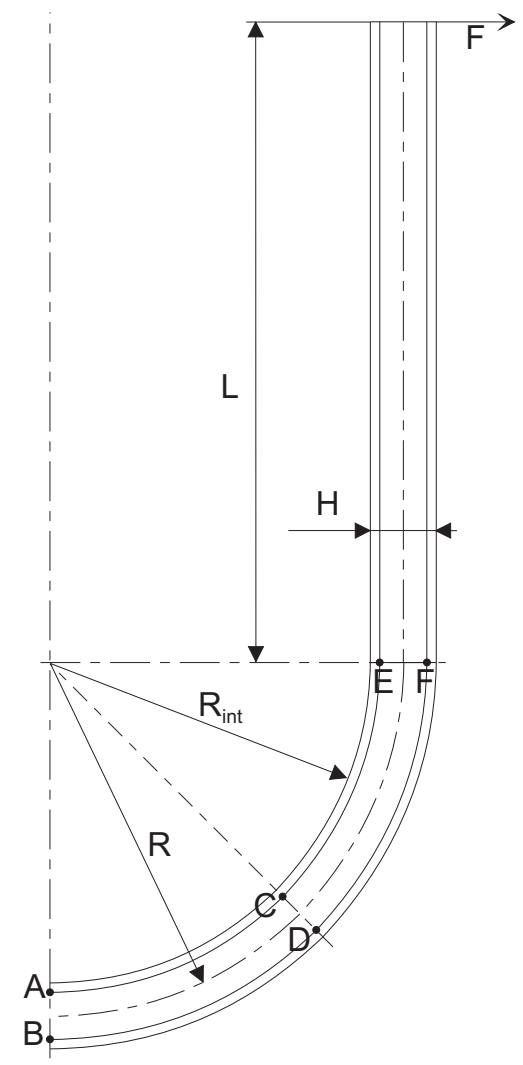

Fig. 5. U-type beam

$70 \mathrm{MPa}$ (soft foam). The applied lateral force is $F=1 \mathrm{~N} / \mathrm{mm}$ (the thickness of the beam is equal to $1 \mathrm{~mm}$ ). By symmetry, only one half of the beam has been modelled.

This example is intended to prove the stability of local Reissner method when the ratio $R / H$ becomes small at the same time that the ratio of Young moduli $E_{s} / E_{c}$ becomes higher (see figure 4 ).

The meshes used are the following:

- reference: 2 elements through the thickness of each skin, 16 through the thickness of the core, nrcuts longitudinal cuts for the inner quarter of circle, $2 \times$ nrcuts for the outer quarter of circle, and ncuts longitudinal cuts for the length $L$.

- computations: 1 element through the thickness of each skin, 2 through the thickness of the core, and nrcuts longitudinal cuts for both inner and outer quarters of circle, and ncuts longitudinal cuts for the length $L$.

The values used for ncuts and nrcuts for the different values of $R / H$ ratio are given in table 1 . They permit to obtain meshes having the same shape as in figure 6 . 
Table 1

parameters of meshes

\begin{tabular}{cc|cc|cc}
$R_{\text {int }}$ & $R / H$ & \multicolumn{2}{|c|}{ reference } & \multicolumn{2}{c}{ computation } \\
& & ncuts & nrcuts & ncuts & nrcuts \\
\hline 5 & 3 & 50 & 20 & 25 & 8 \\
3 & 2 & 50 & 20 & 25 & 8 \\
2 & 1.5 & 50 & 20 & 25 & 6 \\
1 & 1 & 50 & 12 & 25 & 6 \\
0.75 & 0.875 & 50 & 12 & 25 & 6 \\
0.5 & 0.75 & 50 & 12 & 25 & 6 \\
0.25 & 0.625 & 60 & 8 & 30 & 4 \\
0.125 & 0.5625 & 60 & 8 & 30 & 4
\end{tabular}

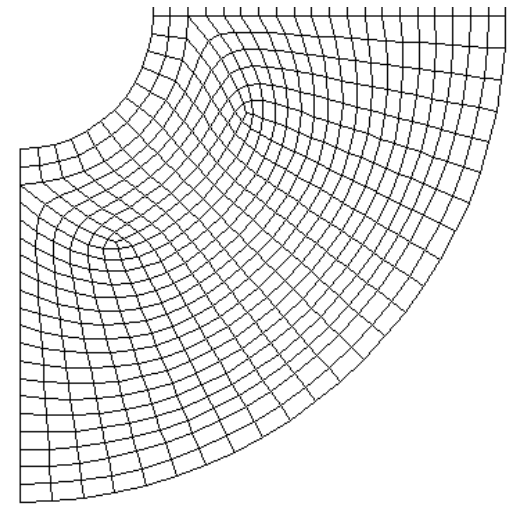

a) reference

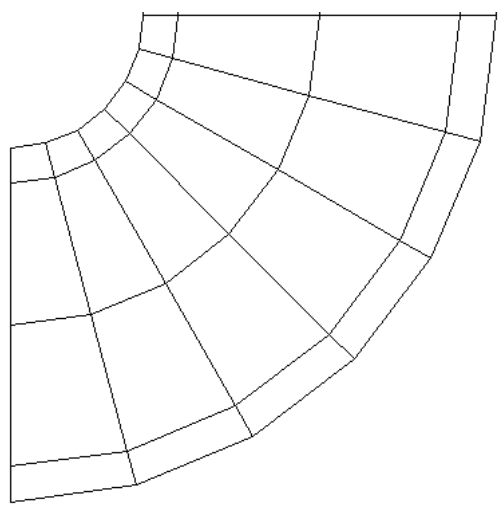

b) computation

Fig. 6. mesh of the quarter of circle (case $\left.R_{\text {int }}=0.75\right)$

We only retain points $A, C$ and $F$, shown in figure 5 , in the results, and we only look at $\sigma_{12}$ or $\sigma_{22}$ (interlaminar stresses: arabic numbers ' 1 ' and ' 2 ' denote local coordinate system whereas ' $\mathrm{x}$ ' and ' $\mathrm{y}$ ' were used for global coordinate system) when both $R / H$ and $E_{s} / E_{c}$ varie.

In the figures presenting the results (figures 7 to 10), the following legend is used: 


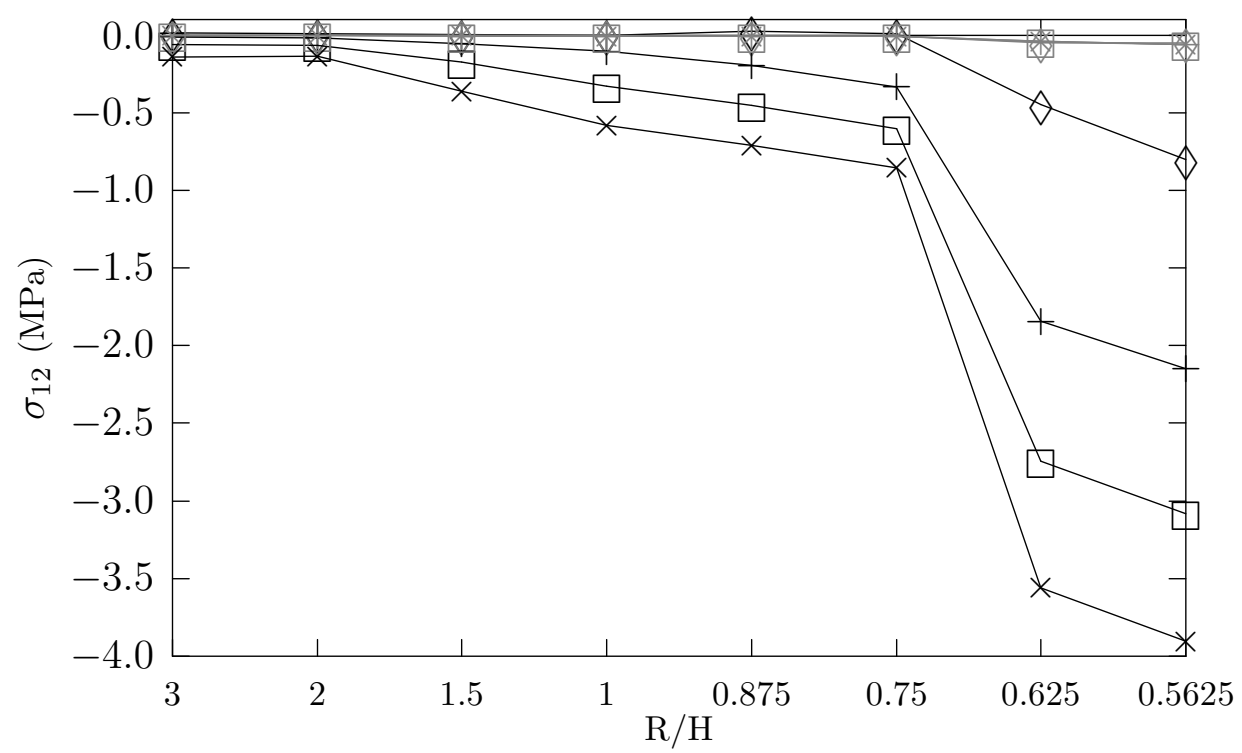

Fig. 7. $\sigma_{12}$ at point $\mathrm{A}$

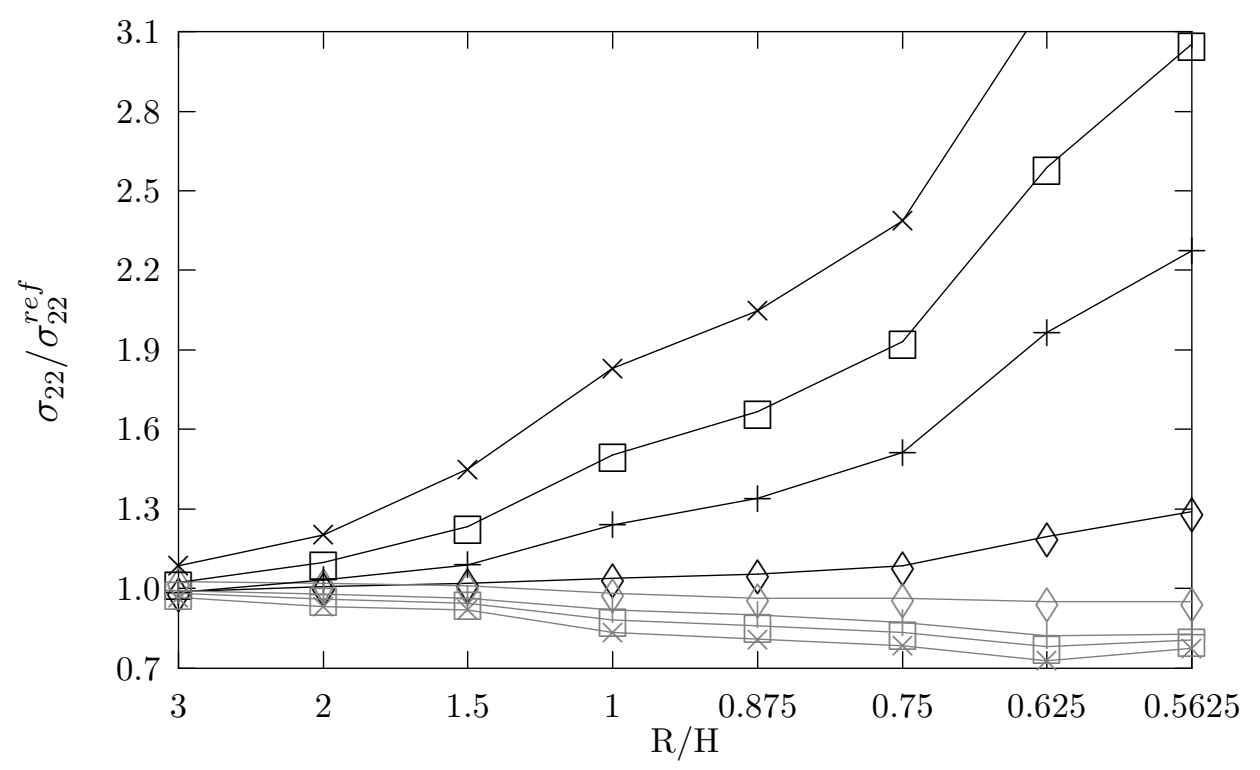

Fig. 8. $\sigma_{22}$ at point $\mathrm{A}$

ANSYs, $E_{s} / E_{c} \approx 20.6 \curvearrowright$

Local Reissner, $E_{s} / E_{c} \approx 20.6$

ANSYS, $E_{s} / E_{c} \approx 206+$

Local Reissner, $E_{s} / E_{c} \approx 206+$

ANSYS, $E_{s} / E_{c}=500 \square$

Local Reissner, $E_{s} / E_{c}=500$

ANSYS, $E_{s} / E_{c}=1000-$

Local Reissner, $E_{s} / E_{c}=1000-x$

Results concerning point $\mathrm{A}$ are given in figures 7 and $8 . \sigma_{22}$ at point $\mathrm{C}$ and $\sigma_{12}$ at point $\mathrm{F}$ are reported in figures 9 and 10 respectively. 


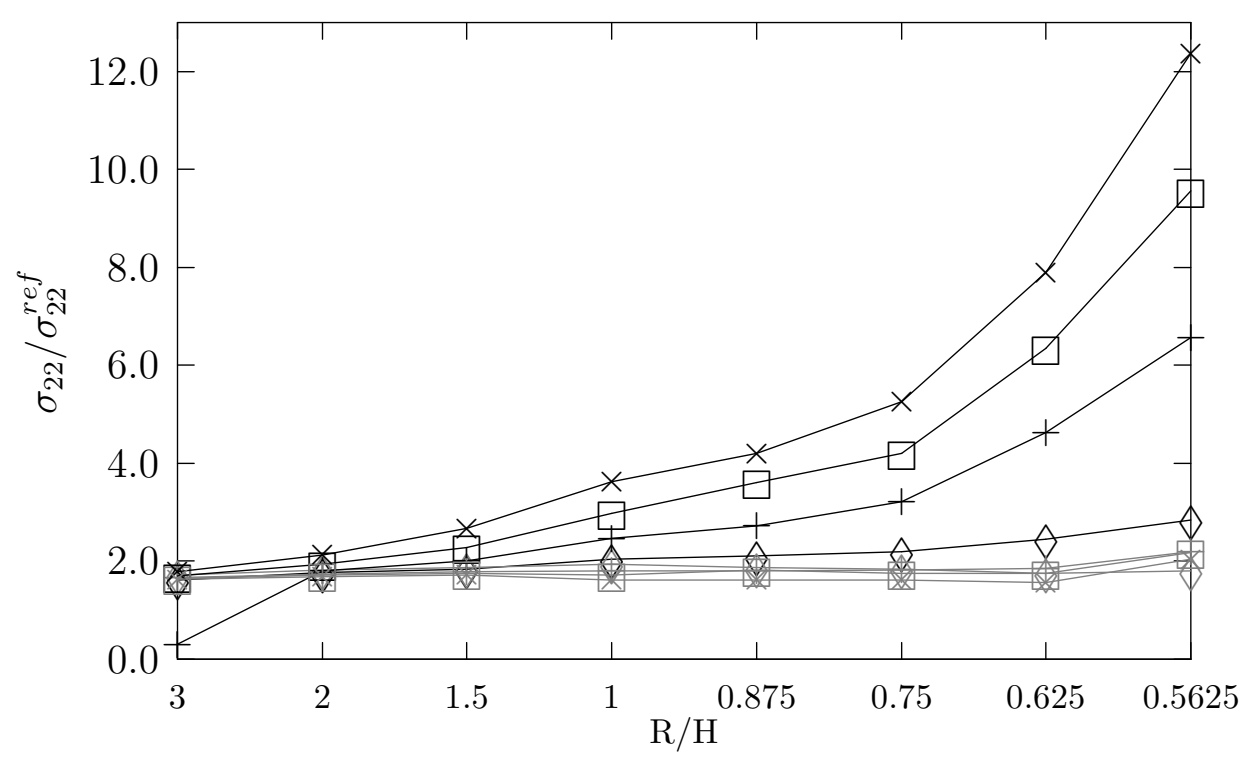

Fig. 9. $\sigma_{22}$ at point $\mathrm{C}$

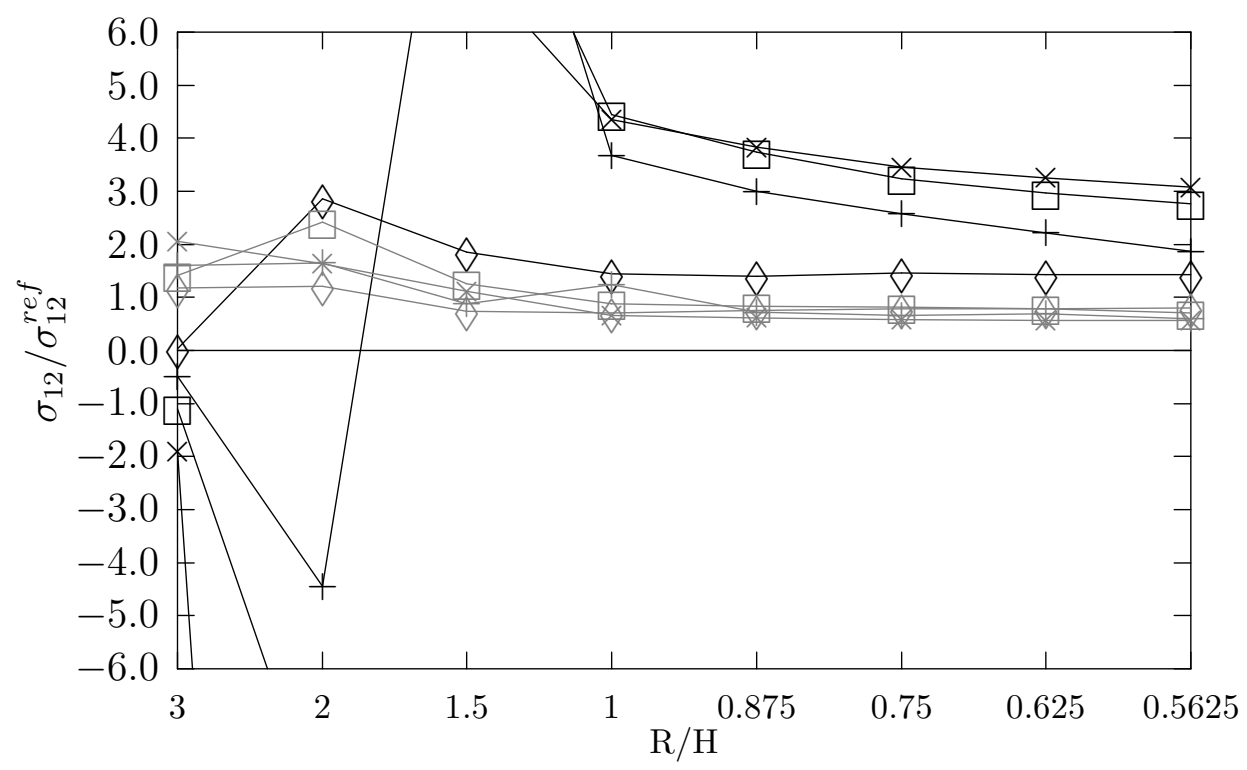

Fig. 10. $\sigma_{12}$ at point $\mathrm{F}$

All these figures (7 to 10) perfectly illustrate the fact that the local Reissner method is not sensitive to the singularity.

- Figure 7 shows that $\sigma_{12}$ at point $\mathrm{A}$ is always close to zero, whereas Ansys (least squares and nodal averaging) gives values up to $4 \mathrm{MPa}$.

- Figure 8 illustrate the fact that $\sigma_{12} / \sigma_{12}^{r e f}$ at point $\mathrm{A}$ remains close to one, even if it gets a little bit worst when approaching the singularity, whereas Ansys strongly diverges. The same conclusion can be drawn for figure 9 presenting $\sigma_{22} / \sigma_{22}^{r e f}$ at point $\mathrm{C}$.

- Contrary to the others figures, figure 10 presenting $\sigma_{12} / \sigma_{12}^{r e f}$ at point $\mathrm{F}$ ex- 
hibits numerical problems at higher $R / H$ ratios. These problems are not encountered when using the local Reissner method, which gives results remaining close to one.

To conclude this example, we can say that in all cases the local Reissner method:

- leads to more accurate results;

- is quite insensitive to numerical problems accuring with ANSYS.

\section{Conclusions}

In this article, we have seen that the computation of interface stresses can be improved:

- In section 3, we presented the "direct local Reissner" method, which is an alternative to the stress projection method or the least squares method for determining nodal stresses from nodal displacements;

- In section 4, we improved the last method to the "local Reissner" on two elements, yielding more stable and accurate results for stresses, especially at interfaces of multi-layered materials.

The last method, local Reissner, is particularly interesting when dealing with multi-layered materials having very heterogeneous mechanical and geometrical properties, such as sandwiches structures.

From the treated examples, we can conclude that the local Reissner method:

- speed up the convergence (which permits to use coarse meshes);

- is more accurate;

- more stable (quite insensitive to numerical problems).

It is also important to notice its simplicity of formulation and of implementation, even in existing finite element programs.

The last point is that the present formulation can be applied every time that an interface between two different materials is encountered, the method having been shown to be particularly robust when the differences in the mechanical properties between the layers is very important. 
The present method is an interesting tools for engineers involved in the design of structures using any highly-heterogeneous multilayered materials.

\section{References}

[1] R. Teti and G. Caprino, Mechanical behavior of structural sandwiches. in: K.A. Olsonn and R.P. Reichard, eds., Sandwich Construction 1 (Chameleon Press LTD, London, 1989) 53-67.

[2] K. Washizu. Variational methods in elasticity and plasticity. (Pergamon Press, New York, 1982).

[3] S. Aivaz-Zadeh Mokari, Éléments finis d'interface. Application aux assemblages collés et structures stratifiées. PhD thesis, U.T.C., 1984.

[4] V. Manet, Analysis Methods of Interface Stresses in Sandwich Structures by Finite Elements (in french). PhD thesis, university of Clermont-Ferrand, 1998.

[5] V. Manet and W.-S. Han, La modélisation des plaques sandwich par éléments finis hybrides et ses applications, in: B. Peseux, D. Aubry, J.-P. Pelle, and M. Touratier, eds., Actes du troisième colloque national en calcul des structures $\mathbf{2}$ (Presses académiques de l'Ouest, Nantes, 1997) 657-663.

[6] E. Hinton and J.S. Campbell, Local and global smoothing of discontinuous finite element functions using a least squares method. in: Int. J. for Num. Meth. in Eng., 8 (Wiley, New-York, 1974) 461-480.

[7] O.C. Zienkiewicz and R.L. Taylor, The Finite Element Method, Vol. 1 (MacGraw-Hill, London, 1994).

[8] V. Manet, W.-S. Han, and A. Vautrin, Static analysis of sandwich plates by finite elements, in: Proceedings of EUROMECH 360 Colloquium: Mechanics of Sandwich Structures, A. Vautrin ed. (Kluwer academic press, Dordrecht, 1997) 53-60.

[9] N.J. Pagano. Exact solutions for rectangular bidirectional composites and sandwich plates, in: Journal of Composite Materials, 4 (1970) 20-34. 